\title{
Dependence of the acoustoelastic properties and texture of an orthorhombic polycrystalline aggregate on stress (DOI 10.1007/s00707-005-0238-y)
}

J. Lewandowski, Warsaw, Poland

Published online: May 8, 2006 @ Springer-Verlag 2006

1. Equations (1), page 4, line 12 from the bottom should be replaced by

$$
\left\{\left[C(\mathbf{x})_{i j k l}+\sigma^{0}(\mathbf{x})_{i j} \delta_{i k}\right] u(\mathbf{x}, t)_{k, l}\right\}_{, j}=\rho(\mathbf{x}) \frac{\partial^{2} u(\mathbf{x}, t)_{i}}{\partial t^{2}}, \quad i, j, k, l=1,2,3 .
$$

2. Page 5, line 1 from the top:

$\left[\left\langle\delta \sigma^{0}(\mathbf{x})_{i j} \delta u(\mathbf{x}, t)_{k, j}\right\rangle\right]_{, j}$ should be replaced by $\left[\left\langle\delta \sigma^{0}(\mathbf{x})_{i j} \delta u(\mathbf{x}, t)_{k, l}\right\rangle\right]_{, j}$

3. Equations (3), page 5, line 12 from the bottom should be replaced by

$$
\left\{\left[\left\langle C(\mathbf{x})_{i j k l}\right\rangle+\left\langle\sigma^{0}(\mathbf{x})_{i j}\right\rangle \delta_{i k}\right]\left\langle u(\mathbf{x}, t)_{k, l}\right\rangle\right\}_{, j}=\langle\rho(\mathbf{x})\rangle \frac{\partial^{2}\left\langle u(\mathbf{x}, t)_{i}\right\rangle}{\partial t^{2}}, \quad i, j, k, l=1,2,3 .
$$

4. Page 5, line 19 from the top:

" $\langle\mathbf{u}(\mathbf{x}, t)\rangle "$ should be replaced by " $\langle\mathbf{u}(\mathbf{x}, t)\rangle, "$

5. Page 11, line 5 from the bottom:

"caculations" should be replaced by "calculations"

6. Page 13, line 3 from the bottom:

".denote" should be replaced by "denote"

7. Page 14, line 13 from the bottom:

"and $n_{\phi}\left(\xi_{1}, \xi_{2} ; \sigma_{11}^{0}=\sigma_{m}\right)$ " should be replaced by "and $n_{\theta}\left(\xi_{1}, \xi_{2} ; \sigma_{11}^{0}=\sigma_{m}\right)$ ",

8. Page 14, lines 12 and 11 from the bottom:

" $[0,360]$ " and " $[0,180]$ " should be replaced by " $\left[0^{\circ}, 360^{\circ}\right]$ " and " $\left[0^{\circ}, 180^{\circ}\right]$ ", respectively.

9. Page 14, line 10 from the bottom:

"size of 18 " should be replaced by "size of $18^{\circ}$ "

10. Page 15, line 6 from the bottom:

" $\Delta n \check{\theta}$ " should be replaced by " $\Delta n_{\theta}$ "

11. Page 16 in Table 1:

" $n_{\varphi}$ and $\Delta n_{\dot{\phi}}$ " should be replaced by " $n_{\varphi}$ and $\Delta n_{\varphi}$ "

12. Page 16, line 20 from the bottom:

" $C_{11}, C_{12}, C_{44}$ " should be replaced by " $c_{11}, c_{12}, c_{44}$ " 
13. Page 18, line 2 from the top:

" $\sigma_{i j}^{0}=n \cdot \Delta \sigma_{i j}^{0}$ " should be replaced by " $\sigma_{i j}^{0}=n \cdot \Delta \sigma_{i j}^{0}$ " and

"N. $\Delta \sigma_{i j}^{0}$ " should be replaced by " $N \cdot \Delta \sigma_{i j}^{0}$ "

14. Page 18, lines $18-21$ from the top:

The sentence:

The Voigt approximation is found to give good agreement with experiment and appears to be the most useful approach for the calculation of ultrasonic velocities in highly textured materials.

should be placed between quotation marks as written literally after [18, p. 555].

15. Page 18, lines 23, 21, 20, 18 from the bottom:

" $v_{i j}$ " should be replaced by " $V_{i j}$ "

16. Page 19, line 1 from the bottom:

" $\Delta n_{\check{\phi}}, \Delta n_{\check{\theta}}$ " should be replaced by " $\Delta n_{\phi}, \Delta n_{\theta}$ " 